\title{
Prácticas de participación política en línea de jóvenes latinoamericanos
}

\author{
Online political participation practices of Latin American youth \\ Carlos Arturo Torres Gastelú
}

\begin{abstract}
RESUMEN
Estudio de percepción sobre las prácticas de participación política en línea que ejercen los jóvenes cuando están conectados a la Internet. En el documento se muestran las similitudes y diferencias en la percepción de los estudiantes universitarios y entre grupos de países. Se optó por un abordaje cuantitativo mediante la aplicación de una encuesta integrada por 16 ítems. En el estudio participaron 2,810 estudiantes universitarios latinoamericanos (1,713 de México, 651 de Colombia y 446 de Perú). Se realizó el análisis de datos mediante la estadística descriptiva e inferencial. Los resultados señalan que existen diferencias significativas en las percepciones de los jóvenes hacia las prácticas de participación política en línea tanto en las formas de activismo político que realizan en la Internet como en sus actitudes hacia el rol de la participación en línea. Sin embargo, en la comparativa entre grupos de países se detectaron similitudes en la mayoría de los ítems relacionados con el activismo político en Internet. Referente a la actitud de los jóvenes universitarios hacia el rol de la participación en línea no se encontraron similitudes entre los países de México,

ABSTRACT

Perception study on online political participation practices carried out by young people when connected to the Internet. The document shows the similarities and differences in the perception of university students, and between groups of countries. A quantitative approach was chosen through the application of a survey made up of 16 items. The study involved 2,810 Latin American university students (1,713 from Mexico, 651 from Colombia and 446 from Peru). Data analysis was performed using descriptive and inferential statistics. The results indicate that there are significant differences in young people's perceptions of online political participation practices, both in the forms of political activism they carry out on the Internet and in their attitudes towards the role of online participation. However, in the comparison between groups of countries, similarities were detected in most of the items related to political activism on the Internet. Regarding the attitude of young university students towards the role of online participation, no similarities were found between the countries of Mexico, Colombia and Peru.
\end{abstract} Colombia y Perú.

Palabras clave: ciudadanía digital, Colombia, México, Perú, universidad.

Keywords: digital citizenship, Colombia, Mexico, Peru, university.

Recibido: julio 13 de 2020 | Aprobado: abril 15 de 2021 | Publicado: mayo 18 de 2021 DOI: https://doi.org/10.33010/ie_rie_rediech.v12i0.950 


\section{INTRODUCCIÓN}

La literatura ha señalado el papel de la participación en línea como un elemento que compone a la competencia de ciudadanía digital (Ferrari, 2013; Carretero, Vuorikari y Punie, 2017). Aunque hay diversas perspectivas de analizar la participación en línea (Choi, 2016; Kara, 2018; Torres-Gastelú, 2020), en este estudio se centra la atención sobre las prácticas de participación política en línea.

En este sentido los gobiernos de los países ejercen un papel relevante para incentivar la activación de la ciudadanía (Escobar, Arana y McCann, 2015), expresado en la responsabilidad de promover en la sociedad el desarrollo de una cultura de participación ciudadana en todos los ámbitos. La creciente inclusión de las tecnologías de la información y la comunicación (TIC) a nivel mundial ha propiciado que los ámbitos para manifestar la ciudadanía se extiendan al campo presencial y al virtual.

Conviene subrayar que la región de América Latina se ha caracterizado por una falta de participación política que se explica por diversos factores como grandes sectores de la población en pobreza, falta de acceso a la educación superior, restricciones socioeconómicas, desconfianza de los individuos hacia las instituciones así como a sus representantes democráticos, entre otros (Gaviria, Panizza y Seddon, 2015).

Además, la región latinoamericana está enfrentando el desafío de la brecha digital, la cual incluye el reto de la mayor cobertura en el acceso a Internet, el desarrollo de las competencias digitales para su adecuada explotación, así como el desafío de la inclusión digital participativa expresada en la asimilación de la competencia ciudadana digital para el ejercicio de sus derechos y obligaciones en la sociedad digital.

Estos aspectos son relevantes considerando que los medios sociales en línea se han constituido como los principales espacios de participación y deliberación pública para grandes grupos poblacionales (De la Garza y Barredo, 2017; De la Garza, Barredo y Arcila, 2017), particularmente en el sector de los jóvenes, cuya identidad se retroalimenta a través de las interacciones en línea que realizan de manera cotidiana.

\section{Revisión de la literatura sobre las prácticas políticas que realizan los jóvenes universitarios de México, Colombia y Perú}

En el caso de Colombia, la participación política se ve afectada por la desigualdad, por los estragos de la violencia y por la invisibilización de los jóvenes. De acuerdo a

Carlos Arturo Torres Gastelú. Académico de la Universidad Veracruzana, México. Es doctor en Ciencias de la Administración, maestro en Ciencias de la Computación y licenciado en Informática. Acumula 32 años de experiencia laboral. Cuenta con reconocimiento al perfil deseable Promep y del Sistema Nacional de Investigadores del Conacyt, Nivel 1. Es miembro del Registro ConACyT de Evaluadores Acreditados (RCEA). Se desempeña como árbitro en diversas revistas internacionales indizadas. Ha publicado 6 libros impresos, 68 capítulos de libros, 56 artículos científicos y 119 artículos en extenso en congresos internacionales. Correo electrónico: torresgastelu@gmail.com. ID: https://orcid.org/0000-0003-2527-9602. 
los reportes de la CEPAL, Colombia sigue siendo uno de los países más desiguales del mundo: unas cuatro de cada diez personas, en el 2013, eran indigentes o estaban en situaciones de pobreza, mucho más marcadas en las zonas rurales que en las zonas urbanas (CEPAL, 2015, p. 61).

En algunas regiones colombianas prevalecen entornos en los que se disuelve la presencia del Estado en favor, por ejemplo, de las mafias asociadas fundamentalmente al narcotráfico, encargadas de establecer las reglas de juego de la convivencia, generando un fenómeno conocido como una "violencia estructural" (Aguilar-Forero y Muñoz, 2015, p. 1023), caracterizado por impactar varios ámbitos como la exclusión de los jóvenes en la participación de la esfera pública colombiana (Patiño, Alvarado y Ospina, 2014), sobre todo en los entornos afectados por el conflicto armado o el narcotráfico (García y Salcedo, 2015).

A raíz de estas circunstancias, en algunas zonas del país es común que los jóvenes a menudo sean invisibilizados en aspectos tales como: asociación injustificada con episodios delincuenciales; falta de inclusión en la generación de consensos por parte de las instituciones; falta de oportunidades laborales adecuadamente remuneradas (Aguilar-Forero y Muñoz, 2015, p. 1023), así como la falta de información, sin un mayor contraste o profundidad (Cárdenas-Ruiz, 2016).

A pesar de ello, los jóvenes colombianos han demostrado su capacidad de protesta juvenil por medio de la tecnología, como fue la movilización del 2011 contra la Ley 30 impulsada por el presidente Santos (Parra y Pinzón, 2015). En dicha protesta los jóvenes se activaron contra la propuesta de reformar una reglamentación del acceso a la educación superior marcado por la privatización del servicio (Galindo, 2016).

Esa práctica de participación política en línea revela una emancipación liberadora mediante la resignificación de su valía y la de otros a través de su posibilidad de expresar sus puntos de vista (Patiño, Alvarado y Ospina, 2014, p. 274). Este mecanismo de manifestación en línea permitió generar una deliberación colectiva al activar la participación desde numerosos mecanismos, como foros, chats, portales informativos, redes sociales, entre otros (Orozco y Ortiz, 2014, p. 92).

En el caso de México, Padilla y Flores (2011) analizaron las prácticas políticas que realizan los jóvenes universitarios utilizando como soporte mediático la Internet, con relación a las instancias políticas formales. Estos autores consideran que la naturaleza de Internet y lo político propiciará un horizonte democrático, aunque existen riesgos latentes como la delincuencia, la vigilancia y el control. Reconocen que las actuales prácticas de los jóvenes no se centran solo en Internet. A pesar de ello, ha favorecido la conformación de comunidades digitales con diversos propósitos. En este mismo orden de ideas, años más tarde, Padilla (2014) encontró que las prácticas políticas que realizan los jóvenes en el estado mexicano de Aguascalientes se caracterizan por expresar sus posturas y reflexiones pero sin acciones o resultados concretos. 
De la misma manera, en otro estudio similar se buscó conocer los hábitos y perspectivas de los estudiantes universitarios de la Ciudad de México en torno a la participación política, la libertad de expresión y el uso de redes sociales. Los investigadores señalan que las redes sociales fungen solo como espacios de circulación informacional y exposición a flujos de datos, y no tanto como herramientas para participar (Meneses, Ortega y Urbina, 2013).

A pesar de ello, la revisión de la literatura en el contexto mexicano muestra evidencias de las prácticas políticas en línea que han redituado en movimientos de estudiantes favorecidos por las redes sociales. A manera de ejemplo se encuentran los casos de \#YoSoy132 (Meneses, 2015) y \#TodossomosAyotzinapa (Meneses y Castillo, 2016). Estos dos movimientos son señales de la forma en que las redes sociales digitales se conforman como espacios que ofrecen oportunidades para la participación política juvenil (Galindo y Gonzáles-Acosta, 2013; Meneses, Ortega y Urbina, 2014).

Sin embargo, en el estudio realizado por Ribeiro, Neves y Menezes (2017) destacan que existen signos de apatía política y desconfianza en la sociedad mexicana, con un especial énfasis en la juventud. A pesar de que las redes sociales propician una ruta alterna confiable para que los jóvenes se expresen, dialoguen y se movilicen (Meneses, Ortega y Urbina, 2017), aún falta mucho camino por recorrer para propiciar que estos canales tecnológicos se conviertan en un efectivo mecanismo para que los jóvenes manifiesten sus opiniones de manera espontánea y abierta.

En este sentido, resulta interesante conocer cuáles son sus intereses y qué podrían aportar a la actividad política tradicional o bien al activismo político en línea. En este tenor, el estudio de Padilla (2013) se dio a la tarea de analizar a los jóvenes y la participación política en Internet. Para ello propuso un nivel de subactivismo en el que pueden darse "posibilidades de análisis de la relación entre un tipo de práctica política -como la que promueven los jóvenes en Facebook con temas de discusión sobre los problemas sociales, económicos y políticos- que puede abonar en la formación ciudadana de los participantes" (Padilla, 2013, p. 130).

En este estudio, los jóvenes registraron actividades de bajo perfil en las que ellos se limitaban a dar seguimiento y difundir informaciones relacionadas con alguna causa, y menos del 5\% manifestó haber participado en una organización de la sociedad civil o movimientos sociales. Además, en los intercambios cotidianos entre los jóvenes destaca que al menos la mitad de los participantes señalaron la práctica de informarse sobre el acontecer político, gubernamental y/o las campañas políticas. En esta modalidad de participación destacan acciones como leer noticias en línea sobre campañas y gobiernos con $28.5 \%$, recibir mensajes que critican a candidatos durante la campaña con $22.7 \%$, y recibir mensajes que exhortan al compromiso y la civilidad con 15.7\% (Padilla, 2013).

En resumen, los estudios que han abordado las prácticas de participación política en línea de los jóvenes mexicanos destacan: la capacidad de los jóvenes para organi- 
zarse en línea, la difusión de sus ideas a través de diversos dispositivos tecnológicos, la preocupación por transformar las condiciones del entorno y su condición como estudiantes universitarios que los legitima como gremio. Sin embargo, estas prácticas de participación política se enmarcan en un escaso nivel de participación en los jóvenes universitarios (Padilla, 2013; Arias-Cardona y Alvarado, 2015).

En el caso de Perú, el estudio de Barredo-Ibáñez (2013) señala que los medios ejercen un rol principal como mediadores de la realidad en los jóvenes peruanos. Sin embargo, los medios se enfrentan a una grave crisis de credibilidad y de confianza caracterizada por una disminución constante de usuarios. De la misma manera, en el caso peruano persiste tanto la brecha digital como la desigualdad económica, provocando la presencia de barreras de acceso de tipo estructural (Quiroz, 2011).

A pesar de ello, en el Perú, los jóvenes universitarios son protagonistas de movilizaciones de protesta social aún cuando es escasa su pertenencia a organizaciones políticas. Un ejemplo de ello fue el nivel organizativo de los universitarios mediante el uso de redes sociales que se unió a las fuerzas de grupos tradicionales e innovadores en el rechazo a la Ley 30288 del 2014, que establecía un régimen perjudicial para los trabajadores de 18 a 24 años que ingresaban al campo laboral (Fernández-Maldonado, 2015). Este esfuerzo evidencia la manera como las organizaciones juveniles lograron menguar sus diferencias políticas e incluso personales en aras de lograr un bien común mediante el uso de las tecnologías de la información y la comunicación.

En este mismo orden de ideas, en el estudio realizado por Cano-Correa, QuirozVelasco y Nájar-Ortega (2017) encontraron que 92.1\% de los jóvenes consideran que la universidad debería ser un espacio de estudio y aprendizaje para expresar libremente sus opiniones, organizar debates y para la realización de actividades políticas (84,9\%). Un sector de los jóvenes aprueba las diversas formas de actividad política en tanto se relacionen con la reivindicación de sus derechos como estudiantes, la defensa de intereses académicos y la fiscalización del comportamiento de sus autoridades.

Sin embargo, tal como señalan Cano-Correa, Quiroz-Velasco y Nájar-Ortega (2017, p. 76), "si bien los jóvenes universitarios reconocen de manera unánime que las redes sociales posibilitan la comunicación horizontal y la democratización de la opinión, no se constituyen ellos mismos en protagonistas y, más bien, sus prácticas están vinculadas fundamentalmente al consumo". Prueba de ello es que solo 10.1\% de los jóvenes peruanos de universidades públicas manifestó haber participado algunas veces en movilizaciones. En parte, esto se debe al temor que los jóvenes expresaron frente a la violencia que pueden generar las movilizaciones políticas en los espacios públicos.

La revisión de la literatura sobre las prácticas políticas que realizan los jóvenes universitarios de México, Colombia y Perú evidencian la incipiente gestación de las manifestaciones de ciudadanía digital expresadas a través de ciertas prácticas de participación política en línea enmarcados a través del activismo en plataformas digitales 
que, si bien es mesurado, ha jugado un rol dinamizador cuando trasciende el activismo digital o clicktivismo (Karpf, 2010; Halupka, 2018) y propicia las manifestaciones en plazas y espacios públicos (Gerbaudo, 2012; Kavada, 2015; Yang, 2016), denotando el proceso de crecimiento en las identidades juveniles enmarcadas en la presencialidad y virtualidad cotidiana.

\section{ОвJеTIVo}

El objetivo del presente estudio es identificar las prácticas de participación política en línea que realizan los jóvenes universitarios de México, Colombia y Perú.

\section{Metodología}

Este estudio forma parte del proyecto "La ciudadanía digital desde la perspectiva del estudiante universitario", cuyo propósito fue analizar las manifestaciones de ciudadanía digital considerando las siguientes dimensiones: participación y prácticas en línea, seguridad y privacidad en línea, gestión de datos personales y comportamiento ético-tecnológico. Para su estudio la dimensión de participación y prácticas en línea se integró por dos instrumentos. Los resultados de la primera parte del instrumento pueden ser consultados en Torres-Gastelú (2020) sobre la incidencia y frecuencia de participación en línea de los jóvenes universitarios de México, Colombia y Perú. En tanto, en este documento se presentan los resultados de la segunda parte del instrumento, que atiende a las prácticas de participación política en línea que ejercen los jóvenes cuando están conectados a la Internet.

\section{Tipo de estudio, instrumento y muestra}

Se trata de un estudio cuantitativo, con un diseño no experimental transeccional. El tipo de muestreo fue no probabilístico bajo el criterio por conveniencia. Se optó por este muestreo debido a que el investigador tenía un tiempo limitado para llevar a cabo la investigación y limitaciones presupuestarias. En el estudio participaron 2,810 estudiantes universitarios (1,713 de México, 651 de Colombia y 446 de Perú). En tanto, la distribución por sexo indica que 43.6\% de los encuestados fueron hombres y $56.4 \%$ mujeres. El instrumento sobre las prácticas de participación política en línea está compuesto por 16 ítems considerando una escala de Likert con cinco opciones (completamente en desacuerdo, en desacuerdo, indiferente, de acuerdo y completamente de acuerdo). El instrumento originalmente fue elaborado en el idioma inglés por Kara (2018), con una confiabilidad de 0.89 en el Alfa de Cronbach. Sin embargo, para este estudio se optó por replicar la traducción y adaptación de dicho instrumento que fue aplicado a estudiantes de diversas universidades públicas mexicanas (Torres-Gastelú, Cuevas-Salazar, Angulo-Armenta y Lagunes-Domínguez, 
2020). En esa ocasión la confiabilidad del instrumento obtenida mediante el Alfa de Cronbach fue de 0.840 , reflejando una consistencia interna elevada.

\section{Hipótesis}

- Hipótesis 1.- No existen diferencias significativas en las percepciones de los jóvenes universitarios de México, Colombia y Perú hacia las prácticas de participación política en línea (activismo político en Internet, actitud hacia el rol de la participación en línea) que ejercen cuando están conectados a Internet.

- Hipótesis 2.- No existe similitud en las comparaciones realizadas entre los grupos de países referente a las percepciones de los jóvenes universitarios hacia las prácticas de participación política en línea (activismo político en Internet, actitud hacia el rol de la participación en línea) que ejercen cuando están conectados a Internet.

\section{Procedimiento y análisis de datos}

El procedimiento que se siguió para el desarrollo del presente estudio fue el siguiente: (1) Se realizaron estancias cortas de investigación en universidades de México, Colombia y Perú para la aplicación de encuestas de diversos instrumentos para medir la percepción de los jóvenes universitarios hacia las formas de manifestación de su ciudadanía digital. (2) Se contactó a las autoridades de las universidades para explicarles el propósito del estudio; el criterio de selección de la muestra fue que los alumnos estuvieran inscritos en el periodo escolar vigente, y que los profesores permitieran la aplicación de encuestas. (3) Todos los alumnos encuestados participaron por voluntad propia y cuando se les explicó que era un estudio sin fines de lucro, todos ellos dieron su consentimiento. (4) Se aplicó el instrumento en formato impreso y se capturó la información para su análisis. (5) Se utilizó estadística descriptiva e inferencial. Para la comprobación de las hipótesis se ejecutaron diversas pruebas no paramétricas como Anova de un factor, comparaciones múltiples post hoc con el método Tukey con el programa estadístico informático para las ciencias sociales (SPSS).

El primer cuestionamiento que se aborda es: ¿Cuáles son las diferencias que existen en las percepciones de los jóvenes universitarios mexicanos, colombianos y peruanos? Para responder a esta pregunta se ejecutaron dos pruebas estadísticas, la prueba de comparación de medias ANOva de un factor, así como la prueba no paramétrica de muestras independientes para la comparación de las medianas entre grupos. El segundo cuestionamiento que atiende este documento es: ¿Cuáles son los países que tienen similitudes en las percepciones de los jóvenes universitarios encuestados? Para responder a este cuestionamiento se realizó la prueba estadística sobre las comparaciones múltiples post hoc con el método Tukey. 


\section{Resultados}

\section{Dimensión Activismo político en Internet}

En la figura 1 se grafican los valores obtenidos de la sumatoria de dos escalas (totalmente en desacuerdo y en desacuerdo) sobre la percepción de los jóvenes universitarios de México, Colombia y Perú hacia los ítems de la dimensión Activismo político en Internet. Esta sumatoria llamada "en desacuerdo" aparece para cada uno de los ítems en los tres países. Para una mayor comprensión sobre el comportamiento de las percepciones en los jóvenes, a continuación se hace la descripción completa de los valores de la escala para cada uno de los ítems.

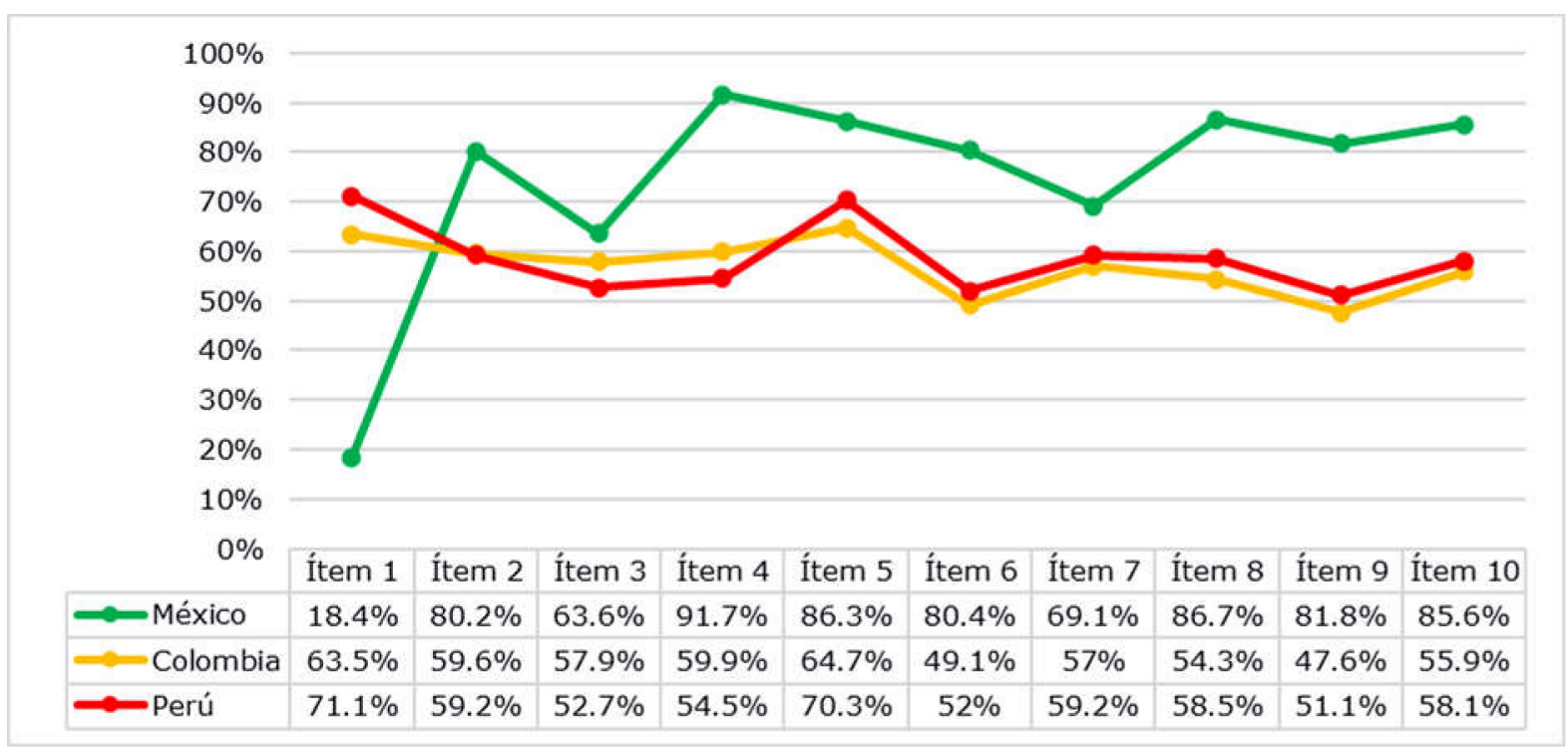

Figura 1. Dimensión Activismo político en Internet por país: datos sobre la escala en desacuerdo.

Fuente: Construcción personal.

Con relación al ítem "Trabajo" o "Soy voluntario para un partido político o candidato de manera virtual mediante Internet”, los jóvenes señalaron que están en desacuerdo con esta aseveración, reflejando una tendencia en al menos dos países: Colombia y Perú (México 18.4\%, Colombia 63.5\% y Perú 71.1\%); mientras que los jóvenes que se mostraron indiferentes a ello se sitúan nuevamente con tendencias porcentuales parecidas entre Colombia y Perú (México 39.2\%, Colombia 22.1\%, Perú 22.4\%). Contrastando con la primera escala, es en México en donde la mayor parte de los jóvenes sí han trabajado o han sido voluntarios virtuales (México 42.4\%, Colombia 14.4\%, Perú 6.5\%).

En tanto para el ítem "Hago publicaciones en línea relacionadas a asuntos políticos o sociales de manera cotidiana”, la opinión de los jóvenes universitarios se distribuye de la siguiente manera: por una parte, se reflejaron mayores porcentajes en los jóvenes de los tres países con un desacuerdo hacia esta práctica en línea (México 
80.2\%, Colombia 59.6\% y Perú 59.2\%); en segundo término aparecen los jóvenes que mantuvieron una postura indiferente sobre este aspecto (México 12.3\%, Colombia $24.3 \%$, Perú $20.4 \%$ ); en tanto, los menores porcentajes se reflejaron en aquellos jóvenes que sí están de acuerdo con esta práctica de participación política en línea (México 7.5\%, Colombia 16.1\%, Perú 20.4\%).

Respecto al ítem "Expreso mis opiniones en línea para desafiar a las perspectivas dominantes o al statu quo con respecto a los asuntos políticos o sociales”, un poco más de la mitad de los jóvenes universitarios de los tres países analizados señaló no estar de acuerdo con este tipo de práctica en línea (México 63.6\%, Colombia 57.9\%, Perú $52.7 \%$ ), en tanto que los jóvenes que se mostraron indiferentes a ello mantienen una similitud en dos países (México 20.9\%, Colombia 27.5\%, Perú 27.1\%), mientras que una pequeña proporción de los jóvenes sostuvo que sí realiza esta práctica en línea (México 15.5\%, Colombia 14.6\%, Perú 20.2\%).

En cuanto al ítem "Pertenezco a grupos en línea que están involucrados en asuntos políticos o sociales", los resultados obtenidos fueron muy precisos en uno de los tres países. En primer término se encuentran los jóvenes que dijeron estar en desacuerdo con realizar esta práctica de participación política en línea, con una tendencia marcada en los mexicanos (México 91.7\%, Colombia 59.9\% y Perú 54.5\%). En segundo término, los jóvenes que adoptaron una postura indiferente para esta aseveración (México 5.2\%, Colombia 22.4\%, Perú 27.4\%). En tercer término se ubican los jóvenes que manifestaron estar de acuerdo en pertenecer a este tipo de grupos en línea (México 3.1\%, Colombia 17.7\%, Perú 18.1\%).

Por otra parte, para el ítem "A veces contacto a funcionarios del gobierno sobre un asunto que es importante para mí mediante métodos en línea”, se observa una tendencia de los jóvenes universitarios a estar en desacuerdo, particularmente por parte de los mexicanos (México 86.3\%, Colombia 64.7\%, Perú 70.3\%); en tanto, no fueron muchos los jóvenes que optaron por mantenerse indiferentes (México 9.1\%, Colombia 21.4\%, Perú 20.9\%); por último, fueron escasos los jóvenes que señalaron estar de acuerdo con este tipo de práctica de participación política en línea (México 4.6\%, Colombia 13.9\%, Perú 8.8\%).

Referente al ítem "Firmo peticiones en línea sobre asuntos sociales, culturales políticos o económicos", los jóvenes de los tres países expresaron una preferencia en no estar de acuerdo con este rubro (México 80.4\%, Colombia 49.1\% y Perú 52\%), mientras que algunos jóvenes se manifestaron indiferentes con este tipo de práctica en línea (México 13.6\%, Colombia 21.4\%, Perú 22.4\%). Por otra parte, un sector de los jóvenes colombianos y peruanos sostuvieron que sí firman peticiones en línea (México 6\%, Colombia 29.5\%, Perú 25.6\%).

Para el ítem "Organizo peticiones en línea sobre asuntos sociales, culturales, políticos o económicos”, más de la mitad de los jóvenes estuvo en desacuerdo con este rubro (México 69.1\%, Colombia 57\%, Perú 59.2\%), en tanto que dos de cada 
diez de los jóvenes expresaron ser indiferentes ante esta aseveración (México 21.2\%, Colombia 24.1\%, Perú 26.5\%). Por último, se concentran los jóvenes que dijeron que sí llevan a cabo esta práctica de participación política en línea (México 9.7\%, Colombia 18.9\%, Perú 14.3\%).

Con relación al ítem "Trabajo en línea con otros para resolver asuntos locales, nacionales o globales", los jóvenes universitarios optaron por estar en desacuerdo (México 86.7\%, Colombia 54.3\% y Perú 58.5\%); en tanto, algunos de los jóvenes adoptaron una postura indiferente para esta situación (México 10.4\%, Colombia $24.7 \%$, Perú $28.5 \%$ ). Con una menor proporción se encuentran los jóvenes que sostuvieron estar de acuerdo con llevar a cabo esta práctica de participación política en línea (México 2.9\%, Colombia 21\%, Perú 13\%).

Para el ítem "Uso Internet con el fin de participar en movimientos/cambios sociales o protestas" es notoria la preferencia de jóvenes mexicanos que dijeron estar en desacuerdo (México 81.8\%, Colombia 47.6\%, Perú 51.1\%). Por su parte, de dos a tres de cada diez de los jóvenes colombianos y peruanos adoptaron una postura indiferente ante este rubro (México 14\%, Colombia 25.8\%, Perú 28\%). De la misma manera, al menos dos de cada diez de los jóvenes colombianos y peruanos señalaron estar de acuerdo con usar Internet para ese fin (México 4.2\%, Colombia $26.6 \%$, Perú $20.9 \%$ ).

Para el ítem "Asisto a reuniones políticas o foros públicos de asuntos locales, municipales o escolares de manera virtual mediante Internet", nuevamente son los jóvenes mexicanos los que se sitúan con una marcada preferencia a estar en desacuerdo (México 85.6\%, Colombia 55.9\% y Perú 58.1\%), aunque de dos a tres de cada diez colombianos y peruanos optaron por adoptar una postura indiferente en este rubro (México 11.4\%, Colombia 25.5\%, Perú 30\%), quedando una escasa preferencia de los jóvenes en asistir a reuniones o foros mediante Internet (México 3\%, Colombia 18.6\%, Perú 11.9\%).

La prueba no paramétrica de muestras independientes para la comparación de las medianas entre grupos arrojó que las medianas de cada uno de los ítems que componen a la dimensión de Activismo político en Internet entre los grupos de estudio (países) difieren entre sí con un nivel de significación del 5\%. Por otra parte, la prueba Anova permitió identificar que los valores en cada uno de los ítems de la dimensión de Activismo político en Internet es menor de 0.05, indicando que las variables están relacionadas y por tanto que hay diferencias significativas entre los grupos (tabla 1). En este caso, el tipo de agrupamiento fue el de país. En la tabla 1 se muestran los valores estadísticos de la media, desviación estándar y F. Cabe señalar que cuanto más alto sea F, más diferencias de medias habrá y por tanto más fuerte es la relación entre las variables. Considerando los resultados de la aplicación de las dos pruebas previas, se concluye que sí existen diferencias significativas en las percepciones de los jóvenes universitarios de México, Colombia y Perú hacia las prácticas de participación política 
en línea relacionada con los ítems que componen a la dimensión de Activismo político en Internet (hipótesis 1).

Tabla 1. Dimensión Activismo político en Internet: estadísticos descriptivos de la prueba ANOVA.

\begin{tabular}{|c|c|c|c|c|c|c|c|}
\hline \multicolumn{2}{|r|}{ Dimensión ítems 1 al 10} & \multicolumn{6}{|c|}{ Concordancia } \\
\hline & Descripción del ítem & País & Media & $\begin{array}{c}\text { Desv. } \\
\text { Std. }\end{array}$ & $\begin{array}{c}\text { Media } \\
\text { cuadrática } \\
\text { (inter-grupos) }\end{array}$ & $\mathrm{F}$ & Sig. \\
\hline \multirow{3}{*}{\multicolumn{2}{|c|}{$\begin{array}{l}\text { 1. Trabajo o soy voluntario para un } \\
\text { partido político o candidato de } \\
\text { manera virtual mediante Internet }\end{array}$}} & México & 3.18 & .878 & \multirow{3}{*}{443.549} & \multirow{3}{*}{467.633} & \multirow{3}{*}{.000} \\
\hline & & Colombia & 2.13 & 1.174 & & & \\
\hline & & Perú & 1.90 & 1.000 & & & \\
\hline & \multirow{3}{*}{$\begin{array}{l}\text { Hago publicaciones en línea } \\
\text { relacionadas a asuntos políticos o } \\
\text { sociales de manera cotidiana }\end{array}$} & México & 1.78 & .932 & \multirow{3}{*}{85.889} & \multirow{3}{*}{81.062} & \multirow{3}{*}{.000} \\
\hline & & Colombia & 2.27 & 1.154 & & & \\
\hline & & Perú & 2.31 & 1.182 & & & \\
\hline \multirow{3}{*}{\multicolumn{2}{|c|}{$\begin{array}{l}\text { 3. Expreso mis opiniones en línea } \\
\text { para desafiar a las perspectivas } \\
\text { dominantes o al statu quo con respecto } \\
\text { a los asuntos políticos o sociales }\end{array}$}} & México & 2.19 & 1.058 & \multirow{3}{*}{12.388} & \multirow{3}{*}{10.591} & \multirow{3}{*}{.000} \\
\hline & & Colombia & 2.27 & 1.117 & & & \\
\hline & & Perú & 2.45 & 1.116 & & & \\
\hline \multirow{3}{*}{\multicolumn{2}{|c|}{$\begin{array}{l}\text { 4. Pertenezco a grupos en línea que } \\
\text { están involucrados en asuntos } \\
\text { políticos o sociales }\end{array}$}} & México & 1.35 & .720 & \multirow{3}{*}{300.682} & \multirow{3}{*}{341.875} & \multirow{3}{*}{.000} \\
\hline & & Colombia & 2.25 & 1.239 & & & \\
\hline & & Perú & 2.37 & 1.145 & & & \\
\hline \multirow{3}{*}{\multicolumn{2}{|c|}{$\begin{array}{l}\text { 5. A veces contacto a funcionarios del gobierno } \\
\text { sobre un asunto que es importante para } \\
\text { mí mediante métodos en línea }\end{array}$}} & México & 1.57 & .837 & \multirow{3}{*}{79.526} & \multirow{3}{*}{86.171} & \multirow{3}{*}{.000} \\
\hline & & Colombia & 2.10 & 1.185 & & & \\
\hline & & Perú & 1.97 & 1.035 & & & \\
\hline \multirow{3}{*}{\multicolumn{2}{|c|}{$\begin{array}{l}\text { 6. Firmo peticiones en línea sobre } \\
\text { asuntos sociales, culturales, } \\
\text { políticos o económicos }\end{array}$}} & México & 1.71 & .914 & \multirow{3}{*}{238.468} & \multirow{3}{*}{205.711} & \multirow{3}{*}{.000} \\
\hline & & Colombia & 2.60 & 1.340 & & & \\
\hline & & Perú & 2.48 & 1.213 & & & \\
\hline & \multirow{3}{*}{$\begin{array}{l}\text { Organizo peticiones en línea sobre } \\
\text { asuntos sociales, culturales, políticos } \\
\text { o económicos }\end{array}$} & México & 1.98 & 1.013 & & & \\
\hline & & Colombia & 2.32 & 1.219 & 32.975 & 28.318 & .000 \\
\hline & & Perú & 2.24 & 1.109 & & & \\
\hline & Trabajo en línea con otros para resolver & México & 1.55 & .794 & & & \\
\hline & asuntos locales, nacionales o globales & Colombia & 2.40 & 1.269 & 212.419 & 226.957 & .000 \\
\hline & & Perú & 2.26 & 1.061 & & & \\
\hline & Uso el Internet con el fin de & México & 1.72 & .857 & & & \\
\hline & participar en movimientos/cambios & Colombia & 2.60 & 1.276 & 238.436 & 232.567 & .000 \\
\hline & sociales o protestas & Perú & 2.50 & 1.125 & & & \\
\hline & Asisto a reuniones políticas o foros públicos & México & 1.56 & .808 & & & \\
\hline & de asuntos locales, municipales o escolares & Colombia & 2.33 & 1.247 & 180.370 & 192.375 & .000 \\
\hline & de manera virtual mediante Internet & Perú & 2.22 & 1.063 & & & \\
\hline
\end{tabular}

El valor de Sig. en cada uno de los ítems fue de .000 
Tabla 2. Dimensión Activismo político en Internet: estadísticos descriptivos de la prueba Tukey.

\section{Descripción del ítem}

1. Trabajo o soy voluntario para un partido político o candidato de manera virtual mediante Internet

2. Hago publicaciones en línea relacionados a asuntos políticos o sociales de manera cotidiana

3. Expreso mis opiniones en línea para desafiar a las perspectivas dominantes o al statu quo con respecto a los asuntos políticos o sociales

4. Pertenezco a grupos en línea que están involucrados en asuntos políticos o sociales

5. A veces contacto a funcionarios del gobierno sobre un asunto que es importante para mí mediante métodos en línea

6. Firmo peticiones en línea sobre asuntos sociales, culturales, políticos o económicos

Organizo peticiones en línea sobre asuntos sociales, culturales, políticos o económicos

8. Trabajo en línea con otros para resolver asuntos locales, nacionales o globales

9. Uso el Internet con el fin de participar en movimientos/cambios sociales o protestas

10. Asisto a reuniones políticas o foros públicos de asuntos locales, municipales o escolares de manera virtual mediante Internet

Subconjunto para alfa $=0.05$

\begin{tabular}{ccccc} 
País & $\mathrm{N}$ & 1 & 2 & 3 \\
\hline Perú & 446 & 1.90 & & \\
\hline Colombia & 651 & & 2.13 & \\
\hline México & 1713 & & & 3.18 \\
\hline Sig. & & 1.000 & 1.000 & 1.000 \\
\hline México & 1713 & 1.78 & & \\
Colombia & 651 & & 2.27 & \\
\hline Perú & 446 & & 2.31 & \\
\hline Sig. & & 1.000 & .729 & \\
México & 1713 & 2.19 & & \\
\hline Colombia & 651 & 2.27 & & \\
\hline Perú & 446 & & 2.45 & \\
\hline Sig. & & .342 & 1.000 & \\
\hline Mexico & 1713 & 1.35 & & \\
\hline
\end{tabular}

\begin{tabular}{cccc} 
México & 1713 & 1.35 & \\
\hline Colombia & 651 & & 2.25 \\
\hline Perú & 446 & & 2.37 \\
\hline Sig. & & 1.000 & .062
\end{tabular}

\begin{tabular}{cccc} 
México & 1713 & 1.57 & \\
\hline Perú & 446 & & 1.97 \\
\hline
\end{tabular}

\begin{tabular}{ccccc}
\hline Colombia & 651 & & & 2.10 \\
\hline Sig. & & 1.000 & 1.000 & 1.000 \\
\hline
\end{tabular}

\begin{tabular}{cccc} 
México & 1713 & 1.71 & \\
\hline Perú & 446 & & 2.48 \\
\hline Colombia & 651 & & 2.60
\end{tabular}

\begin{tabular}{lll}
\hline Sig. & 1.000 & .074 \\
\hline
\end{tabular}

\begin{tabular}{cccc} 
México & 1713 & 1.98 & \\
\hline Perú & 446 & & 2.24 \\
\hline Colombia & 651 & & 2.32 \\
\hline Sig. & & 1.000 & .372
\end{tabular}

\begin{tabular}{cccc} 
México & 1713 & 1.55 & \\
\hline Perú & 446 & & 2.26
\end{tabular}

\begin{tabular}{ccccc}
\hline Colombia & 651 & & & 2.40 \\
\hline Sig. & & 1.000 & 1.000 & 1.000 \\
\hline
\end{tabular}

\begin{tabular}{cccc} 
México & 1713 & 1.72 & \\
\hline Perú & 446 & & 2.50 \\
\hline Colombia & 651 & & 2.60 \\
\hline Sig. & & 1.000 & .147 \\
\hline México & 1713 & 1.56 & \\
\hline Perú & 446 & & 2.22 \\
\hline Colombia & 651 & & 2.33 \\
\hline Sig. & & 1.000 & .081
\end{tabular}


En la tabla 2 se muestran los resultados obtenidos con la prueba de Tukey con objeto de comprobar la hipótesis 2 para la dimensión de Activismo político en Internet. Se visualizan las medias para los grupos en los subconjuntos homogéneos. Se usó la media armónica de los tamaños de grupo (687.754), cabe señalar que los tamaños de grupo no son iguales. Con esta prueba se observa el mismo comportamiento entre los ítems y los países, es decir, es posible identificar a los grupos que mantienen una tendencia similar, reflejando la similitud entre los grupos de países que aparecen en las dos primeras filas de la tabla para cada ítem. Como se observa en la tabla 2, no se encontraron similitudes entre los grupos de los tres países en los ítems 1, 5 y 8 , mientras que en los ítems restantes $(2,3,4,6,7,9$ y 10) sí se detectaron similitudes en la percepción de los jóvenes respecto a los ítems que analizan el activismo político en Internet, en al menos un par de países. De ahí que se concluya que en 7 de los 10 ítems que integran a esta dimensión sí existen similitudes en las comparaciones realizadas entre los grupos de países, sobre las percepciones de los jóvenes universitarios relacionadas con el activismo político en Internet (hipótesis 2).

Cabe señalar que los ítems en los que no se detectaron similitudes entre los grupos de los tres países fueron: (1) “Trabajo o soy voluntario para un partido político o candidato de manera virtual mediante Internet", (5) "A veces contacto a funcionarios del gobierno sobre un asunto que es importante para mí mediante métodos en línea" y (8) "Trabajo en línea con otros para resolver asuntos locales, nacionales o globales".

\section{Dimensión Actitud hacia el rol de la participación en línea}

En la figura 2 se grafican los valores obtenidos de la sumatoria de dos escalas (totalmente en desacuerdo y en desacuerdo) sobre la percepción de los jóvenes universitarios de México, Colombia y Perú hacia los ítems de la dimensión Actitud hacia el rol de la

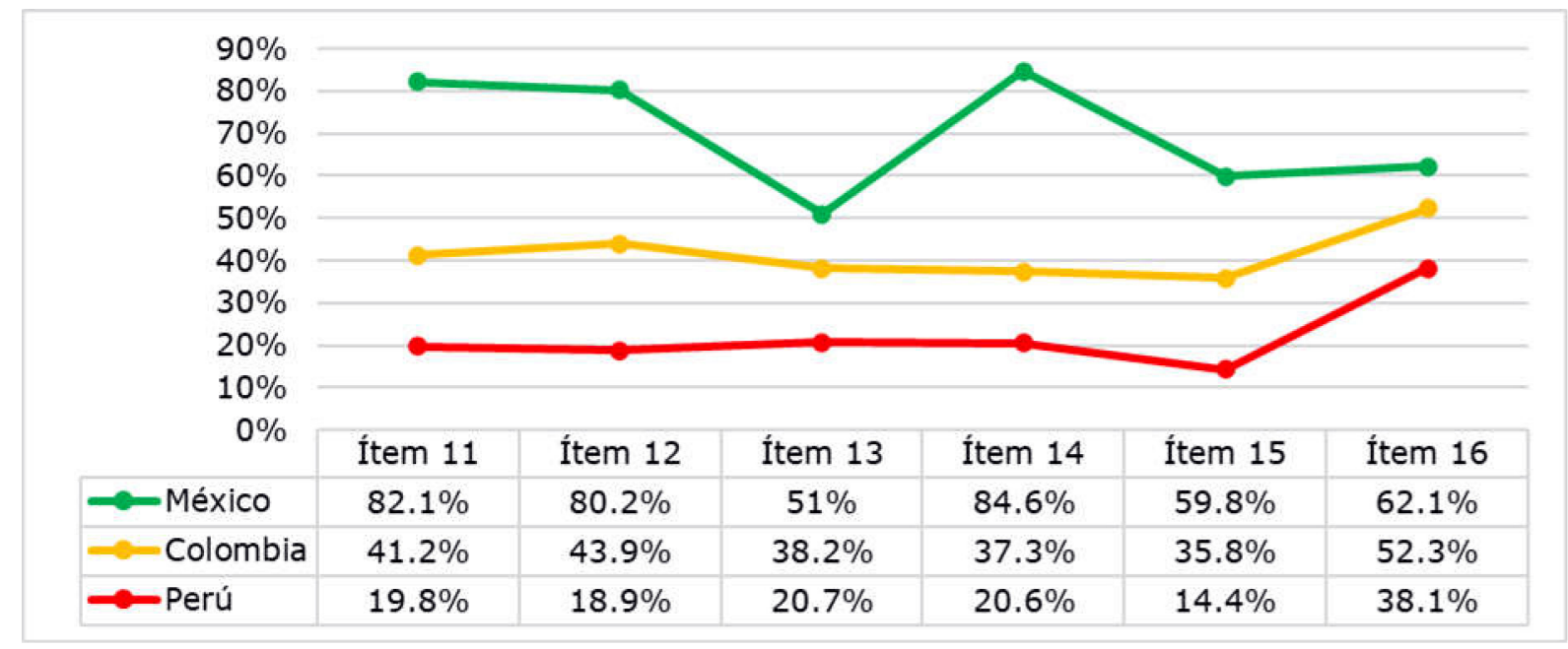

Figura 2. Dimensión Actitud hacia el rol de la participación en línea por país: datos sobre la escala en desacuerdo. Fuente: Construcción personal. 
participación en línea. Esta sumatoria llamada "en desacuerdo" aparece para cada uno de los ítems en los tres países. Para una mayor comprensión sobre el comportamiento de las percepciones en los jóvenes, a continuación se hace la descripción completa de los valores de la escala para cada uno de los ítems.

Respecto al ítem "Considero que la participación en línea es una manera efectiva de participar en asuntos políticos o sociales”, los jóvenes universitarios de dos países señalan estar en desacuerdo con esta aseveración (México 82.1\%, Colombia 41.2\% y Perú 19.8\%), mientras que algunos jóvenes se manifestaron indiferentes con este tipo de práctica en línea (México 14\%, Colombia 23.4\% y Perú 23.8\%), aunque los jóvenes peruanos manifestaron que sí están de acuerdo en considerar efectiva a esta práctica de participación política en línea (México 3.9\%, Colombia 35.4\% y Perú 56.4\%).

En cuanto al ítem "Creo que me ha ocurrido reconsiderar mis creencias respecto a un tema/asunto en particular cuando uso el Internet", los jóvenes de dos países señalaron estar en desacuerdo con esta aseveración (México 80.2\%, Colombia 43.9\% y Perú 18.9\%), de manera más marcada en los mexicanos que en los colombianos, aunque se presentan tendencias un poco más similares en los jóvenes que optaron por manifestarse indiferentes ante este rubro (México 15.6\%, Colombia 27.3\% y Perú 28.3\%). Sin embargo, al menos la mitad de los jóvenes peruanos sostuvieron que están de acuerdo en que alguna vez han reconsiderado sus creencias respecto a un asunto cuando usan el Internet (México 4.2\%, Colombia 28.8\% y Perú 52.8\%).

Con relación al ítem "Considero que la participación en línea promueve el compromiso en el mundo real (fuera de línea)", los jóvenes universitarios que se mostraron en desacuerdo con esta aseveración reflejaron preferencias dispersas (México 51\%, Colombia 38.2\%, Perú 20.7\%), en tanto, entre 2 a 3 de cada 10 jóvenes optaron por una postura indiferente ante este rubro (México 32.1\%, Colombia 29.1\% y Perú $23.5 \%$ ), sin embargo, los jóvenes peruanos indicaron que están de acuerdo en que la participación en línea promueve el compromiso en el mundo real (México 16.9\%, Colombia $32.7 \%$ y Perú 55.8\%).

Referente al ítem "Pienso que la participación en línea es una manera efectiva de hacer cambios a algo que yo creo injusto", los jóvenes mexicanos expresaron una tendencia a estar en desacuerdo ante esta aseveración (México 84.6\%, Colombia $37.3 \%$, Perú $20.6 \%$ ), en tanto, la postura de indiferencia ante esta aseveración se manifestó de manera similar entre los jóvenes (México 10.7\%, Colombia 25.5\% y Perú 22.4\%). Nuevamente, los jóvenes peruanos fueron los que optaron por estar de acuerdo en que la participación en línea es una manera efectiva de hacer cambios a algo que consideren injusto (México 4.7\%, Colombia 37.2\%, Perú 57\%).

En cuanto al ítem "Me parece que el Internet refleja los prejuicios y la presencia dominante de las estructuras de poder fuera de línea”, se acentúa una tendencia en los jóvenes mexicanos que optaron por estar en desacuerdo con respecto a los otros dos países (México 59.8\%, Colombia 35.8\%, Perú 14.4\%). Por otra parte, se presentan 
porcentajes similares en los jóvenes universitarios de los tres países en aquellos que optaron por una postura indiferente hacia esta aseveración (México 26.4\%, Colombia $30.1 \%$, Perú $23.5 \%$ ); en tanto, al menos seis de cada diez jóvenes peruanos señalaron estar de acuerdo en que el Internet refleja los prejuicios y la presencia dominante de las estructuras de poder fuera de línea (México 13.8\%, Colombia 34.1\%, Perú 62.1\%).

Para el ítem "Estoy más comprometido social o políticamente cuando estoy en línea que fuera de línea”, entre 5 y 6 jóvenes colombianos y mexicanos respectivamente optaron por estar en desacuerdo con esta aseveración (México 62.1\%, Colombia 52.3\%, Perú 38.1\%), mientras que se detectaron preferencias parecidas entre los jóvenes de los tres países reflejadas en la adopción de una postura indiferente ante esta situación (México 25\%, Colombia 24.3\%, Perú 35.7\%); sin embargo, las menores preferencias de los jóvenes fueron sobre estar de acuerdo con sentirse más comprometidos social o políticamente cuando están en línea que fuera de línea (México 12.9\%, Colombia 23.4\%, Perú 26.2\%).

La prueba no paramétrica de muestras independientes para la comparación de las medianas entre grupos arrojó que las medianas de cada uno de los ítems que componen a la dimensión de Perspectiva crítica entre los grupos de estudio (países) difieren entre sí con un nivel de significación del 5\%. Por otra parte, la prueba ANOva permitió identificar que los valores en cada uno de los ítems de la dimensión de Perspectiva crítica es menor de 0.05 , indicando que las variables están relacionadas y por tanto que hay diferencias significativas entre los grupos (tabla 3). En este caso, el tipo de agrupamiento fue el de país. En la tabla 3 se muestran los valores estadísticos de la media, desviación estándar y F. Cabe señalar que cuanto más alto sea F, más diferencias de medias habrá y por tanto más fuerte es la relación entre las variables. Considerando los resultados de la aplicación de las dos pruebas previas, se concluye que sí existen diferencias significativas en las percepciones de los jóvenes universitarios de México, Colombia y Perú hacia las prácticas en línea relacionadas con los ítems que componen a la dimensión de Perspectiva crítica (hipótesis 1).

En la tabla 4 se muestran los resultados obtenidos con la prueba de Tukey con objeto de comprobar la hipótesis 2 para la dimensión de Perspectiva crítica. Se visualizan las medias para los grupos en los subconjuntos homogéneos. Se usó la media armónica de los tamaños de grupo (687.754), cabe señalar que los tamaños de grupo no son iguales. Con esta prueba se observa el mismo comportamiento entre los ítems y los países, es decir es posible identificar a los grupos que mantienen una tendencia similar, reflejando la similitud entre los grupos de países que aparecen en las dos primeras filas de la tabla para cada ítem. Como se observa en la tabla 4, no se encontraron similitudes entre los grupos de los tres países en ninguno de los ítems, por tanto, se concluye que no existen similitudes en las comparaciones realizadas entre los grupos de países referentes a las percepciones del joven universitario relacionadas con la perspectiva crítica (hipótesis 2). 
Tabla 3. Dimensión Actitud hacia el rol de la participación en línea: estadísticos descriptivos de la prueba ANOVA.

\begin{tabular}{|c|c|c|c|c|c|c|}
\hline Dimensión ítems 11 al 16 & \multicolumn{6}{|c|}{ Concordancia } \\
\hline Descripción del ítem & País & Media & $\begin{array}{c}\text { Desv. } \\
\text { Std. }\end{array}$ & $\begin{array}{c}\text { Media } \\
\text { cuadrática } \\
\text { (inter-grupos) }\end{array}$ & $\mathrm{F}$ & Sig. \\
\hline \multirow{3}{*}{$\begin{array}{l}\text { 11. Considero que la participación en línea } \\
\text { es una manera efectiva de participar en } \\
\text { asuntos políticos o sociales }\end{array}$} & México & 1.66 & .860 & \multirow{3}{*}{749.594} & \multirow{3}{*}{751.387} & \multirow{3}{*}{.000} \\
\hline & Colombia & 2.89 & 1.280 & & & \\
\hline & Perú & 3.45 & 1.028 & & & \\
\hline \multirow{3}{*}{$\begin{array}{l}\text { 12. Creo que me ha ocurrido reconsiderar } \\
\text { mis creencias respecto a un tema/asunto } \\
\text { en particular cuando uso el Internet }\end{array}$} & México & 1.71 & .877 & \multirow{3}{*}{641.728} & \multirow{3}{*}{641.728} & \multirow{3}{*}{.000} \\
\hline & Colombia & 2.75 & 1.218 & & & \\
\hline & Perú & 3.38 & .973 & & & \\
\hline \multirow{3}{*}{$\begin{array}{l}\text { 13. Considero que la participación en línea } \\
\text { promueve el compromiso en el mundo } \\
\text { real (fuera de línea) }\end{array}$} & México & 2.45 & 1.003 & \multirow{3}{*}{175.866} & \multirow{3}{*}{157.137} & \multirow{3}{*}{.000} \\
\hline & Colombia & 2.87 & 1.182 & & & \\
\hline & Perú & 3.41 & 1.073 & & & \\
\hline \multirow{3}{*}{$\begin{array}{l}\text { 14. Pienso que la participación en línea } \\
\text { es una manera efectiva de hacer } \\
\text { cambios a algo que yo creo injusto }\end{array}$} & México & 1.59 & .859 & \multirow{3}{*}{862.641} & \multirow{3}{*}{899.275} & \multirow{3}{*}{.000} \\
\hline & Colombia & 2.98 & 1.201 & & & \\
\hline & Perú & 3.44 & 1.052 & & & \\
\hline \multirow{3}{*}{$\begin{array}{l}\text { 15. Me parece que el Internet refleja los } \\
\text { prejuicios y la presencia dominante de } \\
\text { las estructuras de poder fuera de línea }\end{array}$} & México & 2.36 & 1.054 & \multirow{3}{*}{289.507} & \multirow{3}{*}{249.307} & \multirow{3}{*}{.000} \\
\hline & Colombia & 2.95 & 1.195 & & & \\
\hline & Perú & 3.57 & .984 & & & \\
\hline \multirow{3}{*}{$\begin{array}{l}\text { 16. Estoy más comprometido social o } \\
\text { políticamente cuando estoy en línea } \\
\text { que fuera de línea }\end{array}$} & México & 2.29 & 1.049 & \multirow{3}{*}{48.562} & \multirow{3}{*}{39.594} & \multirow{3}{*}{.000} \\
\hline & Colombia & 2.55 & 1.256 & & & \\
\hline & Perú & 2.78 & 1.096 & & & \\
\hline
\end{tabular}

El valor de Sig. en cada uno de los ítems fue de .000

Fuente: Construcción personal.

\section{DisCUSIÓN Y CONCLUSIONES}

Las percepciones de los jóvenes latinoamericanos hacia las prácticas de participación política en línea que ejercen cuando están conectados a la Internet fueron analizadas a través de dos dimensiones: las formas de activismo político que realizan en la Internet y las actitudes hacia el rol de la participación en línea.

El análisis sobre las percepciones de los jóvenes latinoamericanos respecto a las formas de activismo político en línea indica que en la mayoría de los ítems al menos siete de cada diez estudiantes universitarios manifestaron que no están de acuerdo con ejercer este tipo de prácticas, aunque hubo marcadas opiniones divididas en cuanto a trabajar o ser voluntarios para un partido político o candidato de manera virtual mediante Internet.

Por otra parte, la actitud hacia el rol de la participación en línea denota que en al menos la mitad de los ítems no manifestaron una actitud positiva. Las perspectivas 
Tabla 4. Dimensión Actitud hacia el rol de la participación en línea: estadísticos descriptivos de la prueba Tukey.

\begin{tabular}{|c|c|c|c|c|c|}
\hline Descripción del ítem & País & $\mathrm{N}$ & 1 & 2 & 3 \\
\hline \multirow{4}{*}{$\begin{array}{l}\text { 11. Considero que la participación en línea es una manera } \\
\text { efectiva de participar en asuntos políticos o sociales }\end{array}$} & Perú & 1713 & 1.66 & & \\
\hline & Colombia & 651 & & 2.89 & \\
\hline & México & 446 & & & 3.45 \\
\hline & Sig. & & 1.000 & 1.000 & 1.000 \\
\hline \multirow{4}{*}{$\begin{array}{l}\text { 12. Creo que me ha ocurrido reconsiderar mis creencias } \\
\text { respecto a un tema/asunto en particular cuando uso el Internet }\end{array}$} & México & 1713 & 1.71 & & \\
\hline & Colombia & 651 & & 2.75 & \\
\hline & Perú & 446 & & & 3.38 \\
\hline & Sig. & & 1.000 & 1.000 & 1.000 \\
\hline \multirow{4}{*}{$\begin{array}{l}\text { 13. Considero que la participación en línea promueve el } \\
\text { compromiso en el mundo real (fuera de línea) }\end{array}$} & México & 1713 & 2.45 & & \\
\hline & Colombia & 651 & & 2.87 & \\
\hline & Perú & 446 & & & 3.41 \\
\hline & Sig. & & 1.000 & 1.000 & 1.000 \\
\hline \multirow{4}{*}{$\begin{array}{l}\text { 14. Pienso que la participación en línea es una manera efectiva } \\
\text { de hacer cambios a algo que yo creo injusto }\end{array}$} & México & 1713 & 1.59 & & \\
\hline & Colombia & 651 & & 2.98 & \\
\hline & Perú & 446 & & & 3.44 \\
\hline & Sig. & & 1.000 & 1.000 & 1.000 \\
\hline \multirow{4}{*}{$\begin{array}{l}\text { 15. Me parece que el Internet refleja los prejuicios y la presencia } \\
\text { dominante de las estructuras de poder fuera de línea }\end{array}$} & México & 1713 & 2.36 & & \\
\hline & Perú & 651 & & 2.95 & \\
\hline & Colombia & 446 & & & 3.57 \\
\hline & Sig. & & 1.000 & 1.000 & 1.000 \\
\hline \multirow{4}{*}{$\begin{array}{l}\text { 16. Estoy más comprometido social o políticamente } \\
\text { cuando estoy en línea que fuera de línea }\end{array}$} & México & 1713 & 2.29 & & \\
\hline & Perú & 651 & & 2.55 & \\
\hline & Colombia & 446 & & & 2.78 \\
\hline & Sig. & & 1.000 & 1.000 & 1.000 \\
\hline
\end{tabular}

Subconjunto para alfa $=0.05$

Fuente: Construcción personal.

de las dos dimensiones permiten vislumbrar indicios sobre el precario desarrollo de la competencia de ciudadanía digital en los jóvenes universitarios latinoamericanos en cuanto a las formas de participación en línea.

En este sentido, se coincide con los hallazgos del estudio realizado por Parra y Pinzón (2015) con respecto al desinterés por los asuntos públicos de los jóvenes universitarios manifestado por una actitud pasiva orientada más hacia la recepción de información, pero que no necesariamente la difunde o interactúa con ella.

En cuanto a las similitudes y diferencias en las percepciones de los jóvenes de los tres países hubo un consenso en que existen diferencias significativas en las percepciones de los jóvenes hacia las prácticas de participación política en línea tanto en las formas de activismo político que realizan en la Internet como en sus actitudes hacia el rol de la participación en línea. Sin embargo, en la comparativa entre grupos de países se detectaron similitudes en la mayoría de los ítems relacionados con el 
activismo político en Internet. Referente a la actitud de los jóvenes universitarios hacia el rol de la participación en línea no se encontraron similitudes entre los países de México, Colombia y Perú.

En siete de los diez ítems se detectaron similitudes en las comparaciones realizadas entre los grupos de países referentes a las percepciones de los jóvenes universitarios relacionadas con el activismo político en la Internet. Los tres aspectos (ítems 1,5 y 8) en los que no se encontraron similitudes fueron: "Trabajo o soy voluntario para un partido político o candidato de manera virtual mediante Internet", "A veces contacto a funcionarios del gobierno sobre un asunto que es importante para mí mediante métodos en línea" y "Trabajo en línea con otros para resolver asuntos locales, nacionales o globales".

En tanto, en las comparaciones realizadas entre los grupos de países referentes a las percepciones de los jóvenes universitarios relacionadas con la actitud hacia el rol de la participación en línea no existen similitudes en ninguno de los ítems, representa un indicio de que a pesar de compartir rasgos comunes en el contexto latinoamericano en aspectos tales como la lengua, ubicación geográfica, antecedentes de dominio extranjero y desarrollo económico en proceso, existen otros factores locales que inciden en la conformación de la identidad digital de los jóvenes universitarios hacia la construcción de las diversas manifestaciones de ciudadanía digital.

En la última década se ha demostrado la capacidad organizativa para manifestarse en línea en aras de un bien común en los tres países en estudio, como el repudio a la injusticia social o bien el rechazo hacia un partido político en época electoral en México (Meneses, 2015; Meneses y Castillo, 2016), la protesta juvenil por medio de la tecnología contra una propuesta de reformar a una ley en Colombia (Parra y Pinzón, 2015; Galindo, 2016) y el nivel organizativo de los universitarios mediante el uso de redes sociales en rechazo a una ley en el Perú (Fernández-Maldonado, 2015). Sin embargo, el desarrollo de la competencia de ciudadanía digital en los jóvenes es incipiente en virtud del escaso interés que señalaron en la mayoría de las diversas formas de ejercer las prácticas de participación política en línea.

A raíz de los resultados obtenidos en este estudio, se recomienda la incorporación de cursos formativos que sean transversales a todos los programas educativos para el nivel universitario, cuyo diseño se oriente en incluir el desarrollo de actitudes, habilidades y conocimientos en los jóvenes para desempeñarse como ciudadanos digitales en una sociedad que demanda el pleno ejercicio de sus derechos hacia las prácticas de participación en línea, así como sus responsabilidades y obligaciones sobre las implicaciones de las distintas manifestaciones y publicaciones que realizan.

Por último, se señalan algunas limitaciones del estudio. En primer lugar, el instrumento se orientó a determinar la percepción de los estudiantes universitarios, por lo tanto, en trabajos futuros se podría incorporar la medición de las habilidades y conocimientos que los jóvenes poseen para realizar dichas actividades. En segundo 
lugar, el tamaño de la muestra que se aplicó en las universidades de los tres países participantes estuvo limitada a la capacidad del investigador para el levantamiento de las encuestas por cuestiones de tiempo y presupuestales, de ahí que la aplicación del instrumento a muestras estadísticamente significativas permitiría corroborar los resultados de este estudio.

\section{REFERENCIAS}

Aguilar-Forero, N., y Muñoz, G. (2015). La condición juvenil en Colombia: entre violencia estructural y acción colectiva. Revista Latinoamericana de Ciencias Sociales, Niñezy Juventud, 13(2), 1021-1035. https:// doi.org/10.11600/1692715x.13233090913.

Arias-Cardona, A. M., y Alvarado, S.V. (2015). Jóvenes y política: de la participación formal a movilización informal. Revista Latinoamericana de Ciencias Sociales, Niñezy Juventud, 13(2), 581-594. http://dx.doi.org/1 $0.11600 / 1692715 x .1322241014$.

Barredo-Ibáñez, D. (2013). La crisis de credibilidad de las organizaciones periodísticas: hacia la sociedad de los ideantes. Poliantea, 9(16), 101-126. https://doi. org/10.15765/plnt.v9i16.395.

Cano-Correa, A. M., Quiroz-Velasco, M. T., y NájarOrtega, R. (2017). Jóvenes universitarios en Lima: política, medios y participación. Revista Comunicar, 53(25), 71-79. https://doi.org/10.3916/C53-2017-07.

Cárdenas-Ruiz, D. J. (2016). Cubrimiento mediático de las elecciones locales de 2015 en Colombia: entre la opacidad política y el centralismo informativo. Paper Político, 21(2), 319-324. https://doi.org/10.11144/ Javeriana.papo21-2.cmel.

Carretero, S., Vuorikari, R., y Punie, Y. (2017). DIGCOMP 2.1 The digital competence framework for citizens. With eight proficiency levels and examples of use. Luxemburgo: Publications Office of the European Union. https:/ / doi.org/10.2760/38842.

CEPAL [Comisión Económica para América Latina y el Caribe] (2015). Anuario estadístico de América Latina y el Caribe (Informe Núm. LC/ G.2656-P). CEPAL. Recuperado de: https:/ / repositorio.cepal.org/bitstream/handle $/ 11362 / 39867 /$ S1500739_mu.pdf? sequence $=1$.

Choi, M. (2016). A concept analysis of digital citizenship for democratic citizenship education in the Internet age. Theory \& Research in Social Education, 44(4), 565-
607. https://doi.org/10.1080/00933104.2016.121 0549.

De la Garza, D., y Barredo, D. (2017). Democracia digital en México: un estudio sobre la participación de los jóvenes usuarios mexicanos durante las elecciones legislativas federales de 2015. Index.comunicación, 7(1), 95-114. Recuperado de: https://dialnet.unirioja.es/ descarga/articulo/6277996.pdf.

De la Garza, D., Barredo, D., y Arcila, C. (2017). Participación política y medios de comunicación. Un estudio sobre los jóvenes ecuatorianos. En C. Arcila, D. Barredo y C. Castro (coords.), Comunicación digital: participación y movimientos sociales en América Latina (pp. 93-110). La Laguna, España: Sociedad Latina de Comunicación Social.

Escobar, C., Arana, R., y McCann, J. (2015). Expatriate voting and migrants' place of residence: Explaining transnational participation in Colombian elections. Migration Studies, 3(1), 1-31. https://doi.org/10.1093/ migration/mnt030.

Fernández-Maldonado, E. (2015). La rebelión de los pulpines. Jóvenes, trabajo y política. Otra Mirada.

Ferrari, A. (2013). DIGCOM: A framework for developing and understanding digital competence in Europe. Luxemburgo: Publications Office of the European Union. https:// doi.org/10.2788/52966.

Galindo, J., y González-Acosta, J. I. (2013). \#YoSoy132: la primera erupción visible. Recuperado de: http:/ /www. investigacionucem.com/resources/Galindo_Gonzalez_YoSoy132.pdf.

Galindo, L. (2016). Dinámicas transnacionales en tiempos de Internet: jóvenes, movilización y apropiación de Facebook en Colombia y en Brasil. Desidades, 12(4), 8-16. Recuperado de: http://pepsic.bvsalud. org/scielo.php?script $=$ sci_abstract\&pid $=$ S2318$92822016000300002 \& \operatorname{lng}=\mathrm{es} \& \mathrm{n} r \mathrm{~m}=$ is. 
García, J. R., y Salcedo, L. (2015). La desafección política en Colombia: un análisis sistémico al respecto. Económicas CUC, 36(2), 49-65. Recuperado de: https:// revistascientificas.cuc.edu.co/economicascuc/article/ view/689.

Gaviria, A., Panizza, U., y Seddon, J. (2015). Economic, social and demographic determinants of political participation in Latin America: Evidence from the 1990s. Latin America Journal of Economic Development, 2(3), 151182. https://doi.org/10.35319/lajed.20043279.

Gerbaudo, P. (2012). Tweets and the streets: Social media and contemporary activism. Londres: Pluto Press.

Halupka, M. (2018). The legitimization of clicktivism. Australian Journal of Political Science, 53(1), 130-141. https:// doi.org/10.1080/10361146.2017.1416586.

Kara, N. (2018). Understanding university students' thoughts and practices about digital citizenship: a mixes method study. Educational Technology \& Society, 21(1), 172-185. Recuperado de: https://www.researchgate. net/publication/322223835_Understanding_University_Students'Thoughts_and_Practices_about_Digital_Citizenship_A_Mixed_Methods_Study.

Karpf, D. (2010). Online political mobilization from the advocacy group's perspective: Looking beyond clicktivism. Policy and Internet, 2(4), 7-41. https://doi. org/10.2202/1944-2866.1098.

Kavada, A. (2015). Creating the collective: Social media, the occupy movement and its constitution as a collective actor. Information, Communication \& Society, 18(8), 872-886. https://doi.org/10.1080/1369118X.2015.1043318.

Meneses, M. E. (2015). Ciberutopias: democracia, redes sociales y movimientos red. México: Editorial Porrúa/Tecnológico de Monterrey.

Meneses, M. E., y Castillo, M. C. (2016). \#TodossomosAyotzinapa, storytelling, identidades, representaciones y reflexividad en disputa. Revista de Estudios Culturales de la Universitat Jaume I, 16(2016), 37-56. https://doi. org/10.6035/clr.2016.16.3.

Meneses, M. E., Ortega, E., y Urbina, G. A. (2013). Jóvenes, participación político ciudadana y redes sociales en México 2012. En G. López y E. Tamés (coords.), La libertad de expresión en el proceso electoral de 2012 (pp. 1013). México: PNUD/Coparmex/Itesm-CCM/Porrúa.

Meneses, M. E., Ortega, E., y Urbina, G. A. (2014). Jóvenes conectados y participación político ciudadana en el proceso electoral de México 2012. Revista Versión. Estudios de Comunicación y Politica, (34), 71-92. Recuperado de: https://versionojs.xoc.uam.mx/index. $\mathrm{php} /$ version/article/download/674/670/.

Meneses, M. E., Ortega, E., y Urbina, G. A. (2017). Youth and political participation. \#YoSoy132 and the struggle for freedom of expression in Mexico. Communication \& Society, 30(1), 87-103. https://doi. org/10.15581/003.30.1.87-103.

Orozco, M. M., y Ortiz, A. (2014). Deliberación: actividad política en Internet y redes sociales en Colombia. Panorama, 8(15), 91-100. http://dx.doi. org/10.15765/pnrm.v8i15.552.

Padilla, M. (2013). El sentido de las prácticas políticas de los jóvenes en Internet. Análisis de la EJIPP 2012. En I. Cornejo y L. A. Guadarrama (coords.), Culturas en comunicación: entre la vocación intercultural y las tecnologías de información (pp. 127-152). México: Tintable.

Padilla, M. R. (2014). Ciudadanía política en la red: análisis de las prácticas políticas entre jóvenes universitarios. Comunicación y Sociedad, (21), 71-100. https://doi.org/10.32870/cys.v0i21.572.

Padilla, M. R., y Flores, D. (2011). El estudio de las prácticas políticas de los jóvenes en Internet. Comunicación y Sociedad, (15), 101-122. https://doi.org/10.32870/ cys.v0i15.1141.

Parra, E., y Pinzón, N. J. (2015). Entre la representación y la movilización: escenarios de participación en Colombia (1991-2014). Reflexión Politica, 17(34), 60-73. https://doi.org/10.29375/01240781.2330.

Patiño, J., Alvarado, S. V., y Ospina, M. C. (2014). Ampliación de sentidos sobre las prácticas políticas de jóvenes con vinculación a siete movimientos sociales en Colombia. Revista Latinoamericana de Ciencias Sociales, Niñezy Juventud, 12(1), 257-275. https://doi.org /10.11600/1692715x.12115101012.

Quiroz, M. T. (2011). La televisión: vista, oída y leída por adolescentes peruanos. Comunicar, 18(36), 35-41. https://doi.org/10.3916/C36-2011-02-03.

Ribeiro, N., Neves, T., y Menezes, E. (2017). An organization of the theoretical perspectives in the field of civic and political participation: contributions to citizenship education. Journal of Political Science Education, 13(4), 426-446. https://doi.org/10.1080 /15512169.2017.1354765. 
Torres-Gastelú, C. A. (2020). Participación en línea de los jóvenes en México, Colombia y Perú. Campus Virtuales, 9(1), 69-83. Recuperado de: http://www.uajournals. $\mathrm{com} /$ campusvirtuales/es/revistaes/numeroactual. html?id=251.

Torres-Gastelú, C. A., Cuevas-Salazar, O., AnguloArmenta, J., y Lagunes-Domínguez, A. (2020). Participación en línea: incidencia y frecuencia en estudiantes universitarios mexicanos. El caso de la Universidad Veracruzana. Formación Universitaria, 13(1), 71-82. http://dx.doi.org/10.4067/S071850062020000100071.

Yang, G. (2016). The commercialization and digitization of social movement society. Contemporary Sociology, 45(2), 120-125. https://doi. org/10.1177/0094306116629409.

Cómo citar este artículo:

Torres Gastelú, C. A.(2021). Prácticas de participación política en línea de jóvenes latinoamericanos. IE Revista de Investigación Educativa de la REDIECH, 12, e950. doi: 10.33010/ie_rie_rediech.v12i0.950. 